\title{
Evaluation of Hepatocellular Carcinomas and Liver Metastases- How Far can we Go with Diffusion Weighted Imaging?
}

Gozde Arslan ${ }^{1 *}$ and Ercan Inci ${ }^{2}$

${ }^{1}$ Department of Radiology, Maltepe University, Istanbul, Turkey

${ }^{2}$ Department of Radiology, Bakirkoy Dr.Sadi Konuk Training-Research Hospital, Istanbul, Turkey

\begin{abstract}
Objective: We aimed to determine the usefulness of the apparent diffusion coefficient (ADC) values for the differential diagnosis of hepatocellular carcinomas and most common metastatic liver tumors and we aimed to compare the characteristic properties. We also aimed to detect novel lesions on ADC maps before the lesion is clinically and radiologically detected by conventional methods.
\end{abstract}

Materials and Methods: We evaluated characteristic properties, contrast enhancement patterns of liver metastasis of malignancies like adenocancer, renal cell carcinoma (RCC), gastrointestinal stromal tumor (GIST) and hepatocellular carcinoma (HCC) on magnetic resonance imaging (MRI) and diffusion weighted (DW) imaging. ADCs were measured both from the cystic and solid parts of the tumoral lesions. Solid and cystic parts were grouped seperately and compared individually.

Results: 48 cases, (60\% male and $40 \%$ female) with ages ranging between 48 and 81 were involved. 28 of them had liver metastasis and 20 of them had HCC. MRIs between the years 2009 and 2012 were analyzed. Among mean ADC values measured from solid contrast-enhancing parts, values of the HCC group were significantly higher than the metastasis group $(p=0.004)$. Also, in metastasis group consisting of many distinct primaries, there were differences in ADC values. A retrospective analysis for follow-up cases showed that the ADC values of the parencyhma where a lesion will appear on later follow-up images were lower than the adjacent parenchyma.

Conclusion: We believe that this method may be useful to detect early metastasis. Studies with larger patient groups could give more significant results which would enable diffusion imaging method to be used in this area.

Keywords: Hepatocellular carcinoma; Liver metastases; Diffusionweighted imaging

\section{Introduction}

Liver tumors are divided into two groups as benign and malignant. The most common benign tumor is hemangioma and the most common malignant tumor is metastasis. Metastasis may be originated from many different organs like colon (most commonly), breast, ovary, stomach, pancreas, lungs and kidneys [1]. The most common primary malignant tumor is hepatocellular cancer (HCC) that is mostly seen in cirrhotic patients [2]. While some of these masses were found to be related with metastasis in cases with known primary malignancy, some others' primary is not known and diagnosis is incidental. After the lesion is detected, it is not always easy to find out the primary tumor in cases with metastasis, many examinations are performed to make a diagnosis and besides their financial and emotional load, these examinations lead to unnecessary radiation exposure. Patients are examined with detailed physical examination and laboratory tests, and then depending on the suspected area, chest X-ray, thoracic CT, abdominal Magnetic Resonance Imaging (MRI)/Computer Tomography (CT), colonoscopy, endoscopy, PET-CT and scintigraphy are performed. Biopsies from suspected areas are obtained. In cases thought to have HCC, liver biopsy is performed for definitive diagnosis. Though accurate diagnoses can be achieved by imaging methods like CT and MRI, it is sometimes difficult to find the origin of a single metastatic lesion in a non-cirrhotic liver with discordant clinical findings. In this study, we aimed to compare the characteristic properties on diffusion weighted imaging, contrast enhancement patterns of liver metastasis with various primaries and hepatocellular carcinomas by MRI. We also searched whether a lesion can be detected quantitatively on ADC maps even before it was clinically and radiologically detected.

\section{Method and Material}

In this study, 48 cases, (60\% male and $40 \%$ female) with ages ranging between 48 and 81 were involved. 28 of them had liver metastasis and 20 of them had HCC. The MRIs which were performed between the years 2009 and 2012 were investigated retrospectively. Images were obtained by Siemens Magnetom Avanto 1.5 Tesla, 18 channel MR scanner with phased-array coil. After localizing images were taken in supine position, T1-weighted turbo spin-echo, T1-weighted in phase, out-phase 2D gradient echo, axial breath-hold fat suppressed T2-weighted turbo spin-echo, T2-weighted HASTE (half-fourier echo train length) sequences and on axial plane, diffusion weighted sequences with chemical shift selective fat-suppression technique (TR/ TE, 4100/82); matrix $104 \times 128$ slice numbers 30, section thickness 6 $\mathrm{mm}$, FOV $350 \mathrm{~mm}$, acquisition time $2 \mathrm{~min}$ were obtained by applying gradients on single-shot echo planar sequence in all three directions $(x, y, z)$ on b value of $1000 \mathrm{~s} / \mathrm{mm}^{2}$ in order to sensitize SE sequence to diffusion. PAT factor, 2, PAT mode, parallel imaging with modified sensitivity encoding (m SENSE) was performed. Following DWI, $0.1 \mathrm{~mol} / \mathrm{kg}$ gadopentetate dimeglumine was administered in bolus

*Corresponding author: Gozde Arslan, Department of Radiology, Maltepe University, Istanbul, Turkey, Tel: 9055568808 96; E-mail: gunesgozde@gmail.com

Received December 13, 2017; Accepted December 18, 2017; Published December 24, 2017

Citation: Arslan G, Inci E (2017) Evaluation of Hepatocellular Carcinomas and Liver Metastases- How Far can we Go with Diffusion Weighted Imaging? J Nucl Med Radiat Ther 8: 351. doi: 10.4172/2155-9619.1000351

Copyright: ( 2017 Arslan G, et al. This is an open-access article distributed under the terms of the Creative Commons Attribution License, which permits unrestricted use, distribution, and reproduction in any medium, provided the original author and source are credited. 
Citation: Arslan G, Inci E (2017) Evaluation of Hepatocellular Carcinomas and Liver Metastases- How Far can we Go with Diffusion Weighted Imaging? J Nucl Med Radiat Ther 8: 351. doi: 10.4172/2155-9619.1000351

Page 2 of 4

according to body weight and fat suppressed T1-weighted volume interpolated gradient sequences were obtained on contrast enhanced axial, coronal and sagittal planes.

A senior and a junior radiologist who were blinded to the clinical and pathologic information evaluated the images. The region of interests (ROI) were carefully placed both on the solid and cystic contrast enhancing portions of the tumors. ADC values of solid and cystic parts of all tumoral lesions were measured and compared individually. The mean of the measurements was recorded for each case. In cases with multiple lesions, the largest lesion was selected for measurement. ROIs covered $60-70 \%$ of the lesions. Measurements were repeated about 8 to 10 times for each lesion and mean values were taken. DWI datasets were transferred to an independent workstation (Leonardo console, software version 2.0; Siemens) for post processing and the ADC maps were reconstructed. The ADC values were calculated as follows: $\mathrm{ADC}=$ - $\left[\ln \left(S / S_{0}\right)\right] / b$, where $S$ is the SI of the region of interest (ROI) obtained through 3 orthogonally oriented DWIs or diffusion trace images, $S_{0}$ is the SI of the ROI acquired through reference T2-weighted images, and $b$ is the gradient $b$ factor with a value of $1000 \mathrm{smm}^{2}$. Lesions were defined as either metastasis or HCC according to clinical findings (whether the patient has a known primary or cirrhosis), laboratory findings ( $\alpha$-fetoprotein levels) [3], pathology results (biopsy samples) and/or follow-up imaging methods (identifying the primary lesion of a metastatic focus or a novel metastatic lesion). In all cases, the number of lesions, their appearance in different MRI sequences, contrast enhancement patterns were examined and ADC measurements of solid and cystic parts of lesions were carried out.

\section{Statistical Analysis}

Statistical analyses in this study were performed using NCSS (Number Cruncher Statistical System) 2007 Statistical Software (Utah,
USA) pocket program. Besides descriptive statistical methods (mean, standard deviation) on the assessment of data, independent t-test was used in comparison of paired groups and chi-square test in comparison of qualitative data. The results were assessed at a significance level of $\mathrm{p}<0005$.

\section{Results}

On the result of statistical analysis, 48 cases ranging between ages of 48 and 81, there were no significant difference between Metastasis and HCC groups with respect to the distribution of sex and mean age ( $\mathrm{p}=0.081$ and $\mathrm{p}=0.169$, respectively). Mean age of metastasis group was 58 and HCC group was 53.20. 28 metastasis cases had multiple lesions and 9 of them had single lesion. The largest metastatic lesion was $171 \times 125 \times 134 \mathrm{~mm}$ and the smallest was $62 \times 63 \times 51 \mathrm{~mm}$. Lesions were found on a cirrhotic liver (based on morphological changes, contour abnormalities, nodules) except for the six of the cases which were found on a non-cirrhotic liver and lesions were multiple except for the five of the cases where there was only a single lesion. Largest HCC was $177 \times 174 \times 135 \mathrm{~mm}$ and smallest was $30 \times 20 \times$ $18 \mathrm{~mm}$. HCC group consisted of (according to the order of frequency) metastasis of colon $(n=13)$, pancreas $(n=5)$, lungs $(n=4)$, RCC $(n=2)$,

\begin{tabular}{|c|c|c|}
\hline $\begin{array}{l}\text { Primary of the } \\
\text { Metastasis Group }\end{array}$ & $\begin{array}{l}\text { ADC values (solid parts) } \\
\text { (Median } \pm S D \times 10^{-3} \mathrm{~mm}^{2} / \mathrm{s} \text { ) }\end{array}$ & $\begin{array}{l}\text { ADC values (cystic parts) } \\
\left(\text { Median } \pm \mathrm{SD} \times 10^{-3} \mathrm{~mm}^{2} / \mathrm{s}\right)\end{array}$ \\
\hline Colon & $1.15 \pm 0.13$ & $1.94 \pm 0.36$ \\
\hline Pancreas & $1.12 \pm 0.23$ & $1.56 \pm 0.33$ \\
\hline Breast & 0.99 & - \\
\hline GIST & $1.52 \pm 0.68$ & 2.16 \\
\hline RCC & $1.75 \pm 0.36$ & - \\
\hline Fallopian Tube & 1.9 & - \\
\hline Lung & $0.88 \pm 0.22$ & $1.86 \pm 0.05$ \\
\hline \multicolumn{3}{|c|}{ ADC: Apparent diffusion coefficient } \\
\hline
\end{tabular}

Table 1: Mean ADC values of the metastasis subgroup.

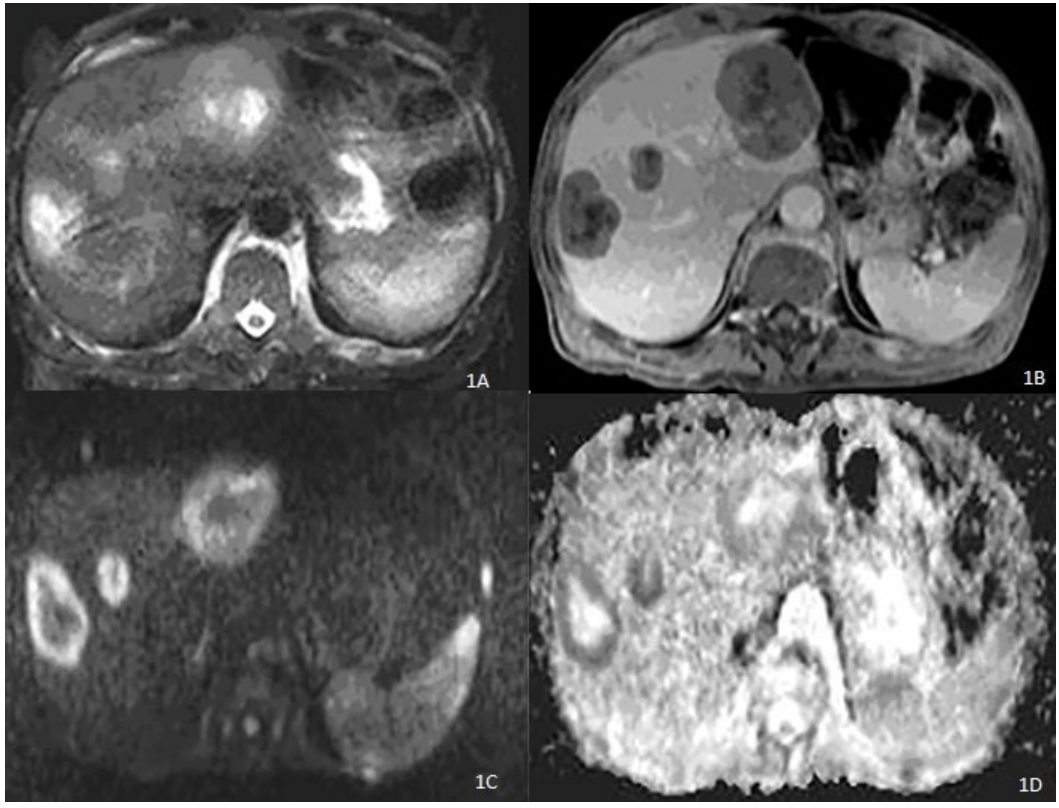

Figure 1: Liver Metastasis of small cell lung carcinoma tumoral foci hyperintense in T2-weighted axial slice (Figure 1A), contrast enhancement in late phase (Figure $1 \mathrm{~B}$ ), solid foci hyperintense in DWI (Figure 1C), and peripherally hypointense, centrally cystic-hyperintense in ADC maps (Figure 1D). Mean ADC of solid parts is 0.724 $\times 10^{-3} \mathrm{~mm}^{2} / \mathrm{s}$. 
Citation: Arslan G, Inci E (2017) Evaluation of Hepatocellular Carcinomas and Liver Metastases- How Far can we Go with Diffusion Weighted Imaging? J Nucl Med Radiat Ther 8: 351. doi: 10.4172/2155-9619.1000351

Page 3 of 4

GIST ( $n=2)$, breast $(n=1)$, tuba ( $n=1)$ (Graphic 1). $90 \%$ of the HCC lesions were hyperintense on T2 weighted images. Only four of them were hypointense. Characteristics on T2-weighted images of metastasis and HCC group did not have significant difference $(p=0.272)$. More than half of hyperintense cases had centrally cystic- necrotic areas. This appearance was observed mostly in metastasis of colon cancer and HCC. Lung cancer metastases were typically target-shaped and GIST metastasis had regular contours on the contrary of other metastasis. Though all kinds of contrast patterns were observed, metastasis group dominantly showed late peripheral contrast enhancement (68\%) and HCC group dominantly showed early peripheral contrast enhancement (60\%). Since solid parts of lesions involve dense tumor cells, significant ADC differences existed between cystic and solid parts. In analysis among all tumors, ADC values of cystic parts ranged between $1.2 \times 10^{-3}$ $\mathrm{mm}^{2} / \mathrm{s}$ and $2.73 \times 10^{-3} \mathrm{~mm}^{2} / \mathrm{s}$ and was mean $1.81 \times 10^{-3} \mathrm{~mm}^{2} / \mathrm{s}$. ADC values of solid parts ranged between $0.72 \times 10^{-3} \mathrm{~mm}^{2} / \mathrm{s}$ and $2.2 \times 10^{-3}$ $\mathrm{mm}^{2} / \mathrm{s}$ and was mean $1.3 \times 10^{-3} \mathrm{~mm}^{2} / \mathrm{s}$ (Table 1 ).

\begin{tabular}{|l|c|c|c|c|}
\hline Variables & Metastasis Group & HCC Group & $\mathbf{t}$ & $\mathbf{p}$ \\
\hline Age & $62.93 \pm 9.25$ & $62.6 \pm 12.97$ & 0.1 & 0.919 \\
\hline ADV Values of Solid Parts & $1.19 \pm 0.34$ & $1.47 \pm 0.27$ & -3.02 & 0.004 \\
\hline ADC Values of Cystic Parts & $1.84 \pm 0.35$ & $1.76 \pm 0.28$ & 0.61 & 0.546 \\
\hline ADC: Appan
\end{tabular}

ADC: Apparent Diffusion Coefficient, MTS: Metastasis, HCC: Hepatocellular Carcinoma

Table 2: Mean ADC values of solid-cystic parts of metastasis and HCC groups.
Statistical analysis of ADC values measured from solid and cystic parts of metastasis and HCC, female- male groups and different age groups had no significant difference. In five cases with small cell lung cancer and breast cancer metastasis, solid region ADC values were 0.862 $\times 10^{-3} \mathrm{~mm}^{2} / \mathrm{s}, 0,723 \times 10^{-3} \mathrm{~mm}^{2} / \mathrm{s}, 724 \times 10^{-3} \mathrm{~mm}^{2} / \mathrm{s}, 0.900 \times 10^{-3} \mathrm{~mm}^{2} / \mathrm{s}$, and $0.959 \times 10^{-3} \mathrm{~mm}^{2} / \mathrm{s}$, respectively. They were low compared to other metastasis. Similar results were reported in different publications [4]. Also measurements of solid regions of RCC and GIST metastasis were mean $1.52 \times 10^{-3} \mathrm{~mm}^{2} / \mathrm{s}$ and $1.75 \times 10^{-3} \mathrm{~mm}^{2} / \mathrm{s}$ respectively and were higher compared to others but, since the number of cases in our study was insufficient, no significant results were obtained (Figure 1).

When metastasis and HCC groups were compared, mean ADC values of cystic regions were $1.84 \times 10^{-3} \mathrm{~mm}^{2} / \mathrm{s}$ and $1.76 \times 10^{-3} \mathrm{~mm}^{2} / \mathrm{s}$ respectively, and statistically insignificant $(\mathrm{p}=0.546)$. Mean $\mathrm{ADC}$ values measured through solid parts were $1.19 \times 10^{-3} \mathrm{~mm}^{2} / \mathrm{s}$ and $1.47 \times 10^{-3}$ $\mathrm{mm}^{2} / \mathrm{s}$, respectively and HCC group values were significantly higher than metastasis group ( $\mathrm{p}=0.004)$ (Table 2).

Besides measurements of followed up patients, new metastatic foci have been detected in metastasis groups. We performed ADC measurements retrospectively and found that diffusion values of parenchymal regions were different from surroundings even when no radiologically detectable lesion existed. We made measurements on the same cases where new foci were diagnosed later on. On 5 follow-up cases with novel metastasis in liver, it was found that in the previous

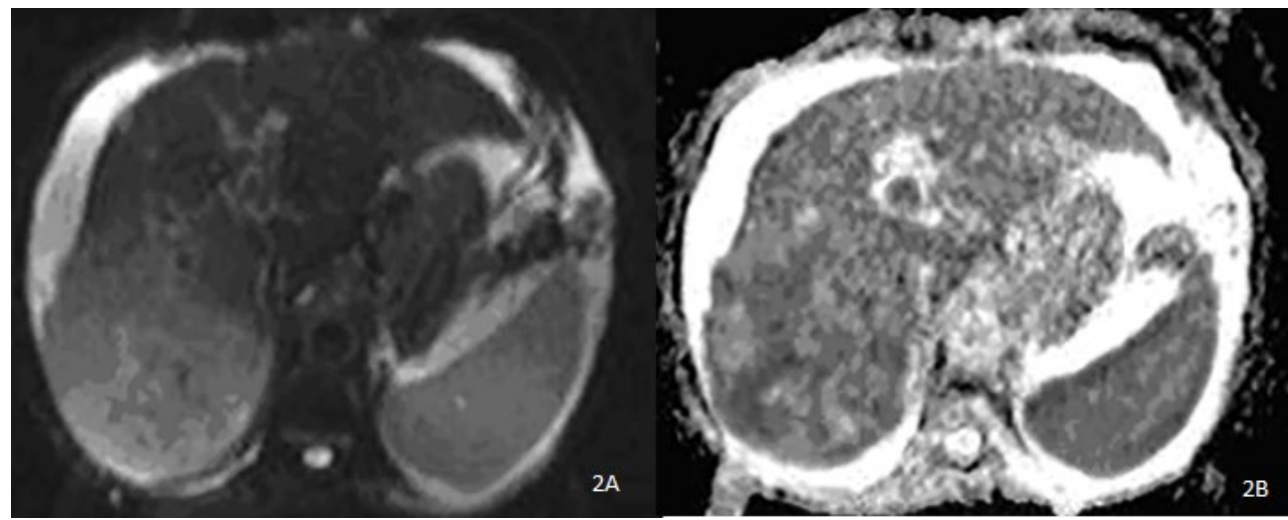

Figure 2: Lesion (Hepatocellular carcinoma) is covering the right lobe posterior segment in a cirrhotic liver. It is hyperintense in T2-weighted images and contrast enhancement is seen in arterial phases. It is hyperintense in DWI sequences (Figure 2A) and hypointense in ADC sequences (Figure $2 B$ ) with mean ADC values of $1.200 \times 10^{-3} \mathrm{~mm}^{2} / \mathrm{s}$.

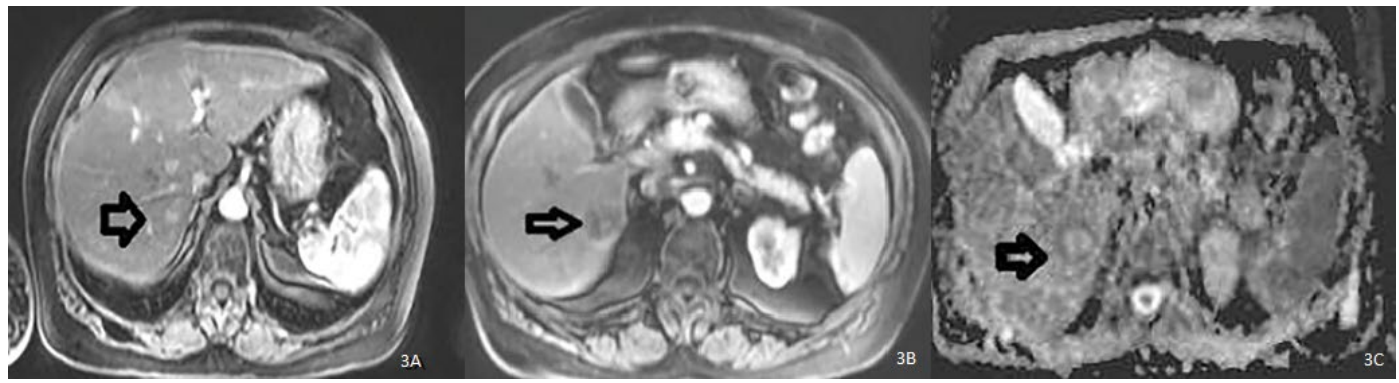

Figure 3: There is no detectable lesion on previous MRI images of a 71-year-old follow-up patient with liver metastasis of breast carcinoma. We measured ADC values from the region where the future lesion will appear (arrow-Figure $3 \mathrm{~A}$ ). Mean ADC was $1.032 \times 10^{-3} \mathrm{~mm}^{2} / \mathrm{s}$. It was low compared to other parts of the liver (mean 1.400 $\times 10^{-3} \mathrm{~mm}^{2} / \mathrm{s}$ ). During follow-ups (Figures $3 \mathrm{~B}$ and $3 \mathrm{C}$ ) we saw a $30 \times 25 \mathrm{~mm}$, contrast enhancing lesion in the right lobe posterior segment of liver on current image. 
(normal) images, ADC values of the lesion-to-be locations were lower compared to adjacent parenchyma (Figures 2 and 3 ).

We believe that this method may be useful to determine metastatic areas that are too small to be detected radiologically. Due to our limited patient number more detailed statistical analysis should be performed in larger group of patients.

\section{Discussion}

Restricted diffusion (high signal intensity in DWI and low signal intensity in ADC) is observed in tissues with high cellularity like tumor and abscess. Contrarily, ADC levels are high in cystic or necrotic tissues $[5,6]$. Attention should be paid to the fact that ADC values may be different in each measurement. Measurement using different devices or even measurement using the same device by the same person may be different in each time. In a study by Sasaki et al., analysis of brain MRI of a group of volunteers showed intra-radiologist difference by 4 to $9 \%$ and inter-radiologist difference by $7 \%$ [7]. In order to prevent this, correct ROI should be chosen and measurements should be repeated when needed. Classical information for liver disease is that multi-slice contrast CT examination is performed in the first plan. Recently, this classical application left its place to MRI. If arterial and venous phase images are obtained with appropriate timing, smaller-sized lesions can be detected on contrast-enhanced dynamic MR images. MRI is also superior for follow-ups. On follow-up for previously detected lesions, a single HASTE sequence is sufficient without using contrast agents. Contrast agents like gadolinium are replaced with hepatospecific contrast agents in some centres. If the aim is to differentiate malignant and benign lesions, RES agents may be preferred, however, in order to differentiate primary malignancies from metastasis, the use of nonspecific extracellular contrast agents may be more useful. Except for these conventional diagnostic methods, methods like diffusion MRI, diffusion tensor MRI, and functional MRI are being used in daily practice. Differential diagnosis of many lesions can be made by the use of diffusion method that measures the movement of water molecules. In previous studies $\mathrm{ADC}$ values have been measured for various pathological conditions in many organs and significant statistical results have been acquired. In the daily practice, the information that acute ischemia causes limited diffusion has been widely used. In our clinic, diffusion weighted images are routinely obtained as well as conventional sequences for brain and abdomen imaging.

In this study, we evaluated characteristic properties, contrast enhancement patterns of liver metastasis of colorectal cancer and various malignancies like adenocancer, RCC, GIST and HCC in differential diagnosis of metastasis in contrast-enhanced MRI and diffusion weighted imaging. We examined and compared ADC measurements. Our aim was to predict the origin of the lesion before biopsy. It is very valuable for both the patient and the physician to find out the primary source of a metastasis in liver by non-invasive methods. Patients undergo many examinations like upper and lower gastrointestinal endoscopy, PET, thoraco-abdominal CT and are exposed to unnecessary biopsies and high dose radiation and may not get satisfactory results even though. In previously-performed, similar studies ADC values of HCC and metastasis have been compared and HCC values have been found to be higher compared to metastasis as in our study $[5,8]$. The difference in our study is that solid and cystic parts of lesions were measured separately in order to prevent the falsepositive results due to elevated ADC values. The mean measured value through the solid parts of all lesions was $1.3 \times 10^{-3} \mathrm{~mm}^{2} / \mathrm{s}$, lower than cystic parts with a mean value of $1.81 \times 10^{-3} \mathrm{~mm}^{2} / \mathrm{s}$. Among mean ADC measurements of solid contrast-enhancing parts, measurements of
HCC group was significantly higher than metastasis group $(\mathrm{p}=0.004)$. There was no significant difference between measurements from cystic parts. In the light of the present and previous studies, differentiating two distinct pathology which have different follow-up protocols and prognosis may be possible by accurate measurements from accurate regions. We found out differences in liver metastasis groups. For example, we found that lung tumor liver metastasis and the sole breast cancer metastasis in our patient group had lower ADC measurements compared to others. However, insufficient number of patients with different histopathological subgroups, which is the main limitation of our study, caused insignificant results. We think that the number of cases with metastasis of different origins should be increased to get significant results.

\section{Conclusion}

Distinguishing primary and metastatic liver tumors (which have different treatment and follow-up protocols) by imaging methods and detection of primary foci of metastatic tumors are of vital importance to decrease unnecessary non-invasive and invasive procedures. HCC and metastasis are not clearly differentiated by classical imaging methods and one of these two diagnoses are estimated according to presence of primary tumor, presence of cirrhotic characteristics, number of lesions and contrast enhancement patterns. Also in metastatic cases with an unknown primary, classical imaging methods are mostly insufficient for differential diagnosis. In this study, we used diffusion weighted MRI method that has been used in many areas and we carried out ADC measurements in lesions of two pathological groups that revealed significant differences. Also in metastasis group with many distinct primary lesions, there were differences in ADC measurements, but the number of our cases was not satisfactory, so we could not get significant results. We performed retrospective analysis in follow-up cases and found that ADC measurements on previous images of new metastatic lesions were different from surrounding tissues which were found to be normal in all sequences including diffusion imaging. This analysis has not been done yet up to our knowledge. We think that this method may be useful to detect early metastases which are invisible by today's imaging methods. But more significant results are needed to be obtained by doing studies with larger group of patients.

\section{References}

1. El-Serag HB, Mason AC (1999) Rising incidence of hepatocellular carcinoma in the United States. N Engl J Med 340: 745-750.

2. Durand F, Regimbeau JM, Belghiti J, Sauvanet A, Vilgrain V, et al. (2001) Assessment of the benefits and risks of percutaneous biopsy before surgical resection of hepatocellular carcinoma. J Hepatol 35: 254-258.

3. Caturelli E, Solmi L, Anti M, Fusilli S, Roselli P, et al. (2004) Ultrasound guided fine needle biopsy of early hepatocellular carcinoma complicating liver cirrhosis: A multicentre study. Gut 53: 1356-1362.

4. Demir OI, Obuz F, Sağol O, Dicle O (2007) Contribution of diffusion-weighted MRI to the differential diagnosis of hepatic masses. Diagn Interv Radiol 13 81-86.

5. Taouli B, Vilgrain V, Dumont E, Daire JL, Fan B, et al. (2003) Evaluation of liver diffusion isotropy and characterization of focal hepatic lesions with two singleshot echo-planar MR imaging sequences: Prospective study in 66 patients. Radiology 226: 71-78.

6. Chan JH, Tsui EY, Luk SH, Fang AS, Wong KP, et al. (2001) Diffusion-weighted MR imaging of the liver: Distinguishing hepatic abscess from cystic or necrotic tumor. Abdom Imaging 26: 161-165.

7. Sasaki $M$, Yamada $K$, Watanabe $Y$, Matsui $M$, Shibata $E$, et al Variability in absolute apparent diffusion coefficient values across different platforms may be substantial: Multivendor, multiinstitutional comparison study. Radiology 2008; 249:624-630.

8. Hosny IA (2010) Diffusion MRI of focal liver lesions. Pak J Radiol 20: 1-7. 